\title{
Experimental characterization of the time correlation properties of nonlinear interference noise
}

\author{
Ori Golani( ${ }^{(1)}$, Daniel Elson, (2), Domanç Lavery ${ }^{(2)}$, Lidia Galdino ${ }^{(2)}$, \\ Robert Killey ${ }^{(2)}$ Polina Bayvel(2), and Mark Shtaif ${ }^{(1)}$
}

(1) School of Electrical Engineering, Tel Aviv University, Tel Aviv, Israel 69978, origolani@post.tau.ac.il

(2) Optical Networks Group, Department of Electronic and Electrical Engineering, UCL, Torrington Place, London WC1E 7JE, UK.

\begin{abstract}
We demonstrate a method for experimentally characterizing the time-evolution statistics of nonlinear interference noise (NLIN). For the first time in an experimental setting strong temporal correlations are observed beyond the phase-noise NLIN component. The ability of measuring these correlation is imperative for designing NLIN mitigation schemes.
\end{abstract}

\section{Introduction}

Modern wavelength division multiplexed (WDM) systems are often limited by nonlinear interference between different WDM channels. From the standpoint of each individual WDM receiver, this interference appears random and is registered as noise. It is often referred to as nonlinear interference noise, or NLIN. In recent years, extensive efforts have been invested into the characterization of NLIN and the exploration of methods for its mitigation. An important finding in this context was that the cross-phase-modulation (XPM) component of NLIN (the predominant component in most practical scenarios) can be modeled very accurately as an inter-symbol interference (ISI) process with random and time varying ISI coefficients ${ }^{1-3}$. Hence, the characterization of NLIN can be translated into the characterization of the corresponding ISI coefficients. Devising reliable experimental methods for doing that is imperative for assessing the prospects of NLIN mitigation and for designing NLIN equalization techniques $^{4-7}$.

Almost all existing research to date has focused on the zeroth-order ISI coefficient, whose contribution to NLIN is phase and polarization rotation noise (PPRN) ${ }^{9-11}$. Yet, in most cases of interest, it is also important to account for higher order ISI terms, whose accumulated effect may exceed the PPRN contribution. One of the obstacles to doing so is the difficulty in evaluating the higher order ISI terms experimentally. While the zeroth-order ISI term produces clear kidney-shaped spots in the received constellation points, the effect of higher order ISI terms is not geometrically obvious, and can be easily confused with additive white noise.

In this paper, we describe a method for measuring general-order ISI coefficients and evaluat- ing their properties. The results validate the importance of the higher-order ISI terms and point to the potential benefits of designing properly tailored equalization methods ${ }^{4}$.

\section{Principal idea}

In this section, we describe the methodology to estimate the statistics of a general-order of ISI coefficient. As demonstrated in Refs. ${ }^{3,8}$, following matched filtering and digital dispersion compensation, the $n$-th sample of the received signal is given by

$$
\left|s^{(n)}\right\rangle=\left|a^{(n)}\right\rangle+\sum_{l=-\infty}^{\infty} \mathbf{R}_{l}^{(n)}\left|a^{(n-l)}\right\rangle+\left|w^{(n)}\right\rangle
$$

where the symbol $|\cdot\rangle$ denotes a two element column vector, accounting for polarization multiplexed transmission. The vector $\left|a^{(n)}\right\rangle$ contains the symbols transmitted in the $n$-th time-slot of the two polarization channels, and $\left|w^{(n)}\right\rangle$ is an additive white noise process that accounts for the effects of amplified spontaneous emission noise and for NLIN generated by FWM. The ISI coefficients $\mathbf{R}_{l}^{(n)}$ are $2 \times 2$ matrices, whose elements are determined by the data transmitted over the interfering WDM channels. It has been demonstrated in $^{1-3}$ that the summation on the righthand-side of (1) accurately accounts for the NLIN that is generated by XPM. The index $l$ in $\mathbf{R}_{l}^{(n)}$ represents the ISI order, whereas the superscript (n) points to the (relatively slow) dependence of the ISI matrices on time. The statistical properties of the ISI matrices have been studied theoretically in ${ }^{3}$. Our goal here is to show how they can be characterized experimentally in practical transmission systems.

In order to better understand the motivation for 
this work, note that as long as the time dependence (i.e. the dependence on $n$ ) of the ISI matrices $\mathbf{R}_{l}^{(n)}$ is sufficiently slow, the effect of XPMinduced NLIN can be undone by means of adaptive linear equalization. Thus, the potential benefit of equalization depends on the variance and temporal correlation length of the ISI matrix elements. For this reason, the experimental assessment of these quantities is of great importance.

In order to extract the properties of $\mathbf{R}_{l}^{(n)}$, we define the quantity

$$
\hat{R}_{l_{j, k}}^{(n)}=\left(s_{j}^{(n)}-a_{j}^{(n)}\right) / a_{k}^{(n-l)},
$$

where $j$ and $k$ each receive one of the values 1,2 , and where $s_{j}^{(n)}$ and $a_{j}^{(n)}$ denote the $j$-th element of $\left|s^{(n)}\right\rangle$ and $\left|a^{(n)}\right\rangle$, respectively. Taking advantage of the whiteness of the process $\left|w^{(n)}\right\rangle$ and of the statistical independence between all transmitted data-symbols, it can be readily shown from (1) that the autocorrelation function (ACF) of the $(j, k)$-th element of $\mathbf{R}_{l}^{(n)}$ is

$$
f_{R_{l, j k}}(\Delta n)=\lim _{N \rightarrow \infty} \frac{1}{N} \sum_{n=1}^{N} \hat{R}_{l_{j, k}}^{(n)} \hat{R}_{l_{j, k}}^{*(n+\Delta n)},
$$

where $\Delta n= \pm 1, \pm 2, \pm 3, \ldots$, and where * stands for complex conjugation. A slightly more involved procedure (also based on Eq. (1)) produces the ACF for $\Delta n=0$. Similar expressions to Eq. (3) can be written for the cross-correlation functions between different elements of $\mathbf{R}_{l}^{(n)}$, or between elements of ISI matrices corresponding to different ISI orders. In the section that follows we describe the measurement of some of these ACF functions. In the experimental results that we present in what follows, we subtract from the ACF of Eq. (2) The square of the time averaged value of $\hat{R}_{l_{j, k}}^{(n)}$ so as to remove unrelated time independent ISI that may be caused by imperfections in filtering, dispersion compensation etc.

\section{Experimental setup and results}

In our experiments, a drive signal was generated from sequences of $2^{16}$ random bits mapped to 64QAM symbols and nonlinearly pre-emphasized using a Volterra filter to compensate for trans-

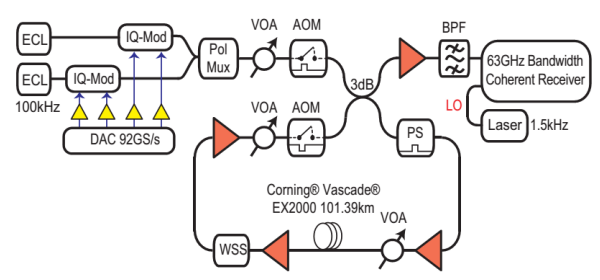

Fig. 1: Experimental setup mitter non-idealities ${ }^{12}$. The symbol rate was 40 GBaud and a Root raised cosine filter with $0.1 \%$ rolloff was applied. The signals were uploaded to a 92 GSa/s DAC, linearly amplified and applied to 2 separate IQ modulators that were used to independently modulate the odd and even WDM channels, which were decorrelated with a 15 ns delay and polarization-multiplexed. The number of WDM channels was 7 , the channel spacing was $42.5 \mathrm{GHz}$, the nominal linewidths were 1.1 $\mathrm{kHz}$ for the central channel and $100 \mathrm{KHz}$ for the other channels.

For transmission, a recirculating loop was used as shown in Fig. 1, with a loop-synchronous polarization scrambler and a span of $101.39 \mathrm{~km}$ of Corning ${ }^{\circledR}$ Vascade ${ }^{\circledR}$ EX2000 fiber with a total loss of $16.2 \mathrm{~dB}$. Two EDFAs (5dB noise figure) separated by a WSS serving as a programmable gain flattening filter were also present inside the loop. The signal was circulated 20 times, reaching a transmission distance of $2020 \mathrm{~km}$. The receiver used an ECL with $12 \mathrm{dBm}$ output power and $1.5 \mathrm{kHz}$ nominal linewidth, and balanced photo-diode detectors. The received signals were captured by a real-time digital oscilloscope with an analogue electrical bandwidth of $63 \mathrm{GHz}$ at $160 \mathrm{GSa} / \mathrm{s}$.

The offline digital processing of the traces included IQ delay compensation, chromatic dispersion compensation, matched filtering, polarization demuxing and equalization. A constant modulus algorithm was applied to preconverge 61 taps for a least mean squares radially directed equalizer. The frequency offset between carrier and LO was estimated before a decision directed algorithm was used for carrier phase estimation using 256 taps. Gram-Schmitt orthogonalization was finally performed to remove sub-optimal phase biasing of the transmitter IQ modulators.

Figure 2 shows the auto-correlation functions for the diagonal matrix elements of the first 5 ISI matrices $l=0, \pm 1, \pm 2$, measured with the proposed method. The auto-correlation functions are analyzed for 3 different signal launch power; $6.5 \mathrm{~dB}$ (linear regime), $-0.5 \mathrm{~dB}$ (optimal launch power) and $5.5 \mathrm{~dB}$ (highly nonlinear regime), as illustrated in Fig. 2 (a),(b) and (c), respectively. The agreement between simulations (black lines) and measurement (coloured lines) is excellent, except in the case of the $l=0$ term, which represents phase and polarization rotation. This discrepancy is expected owing to the combination of laser phase-noise and an adaptive phase recov- 

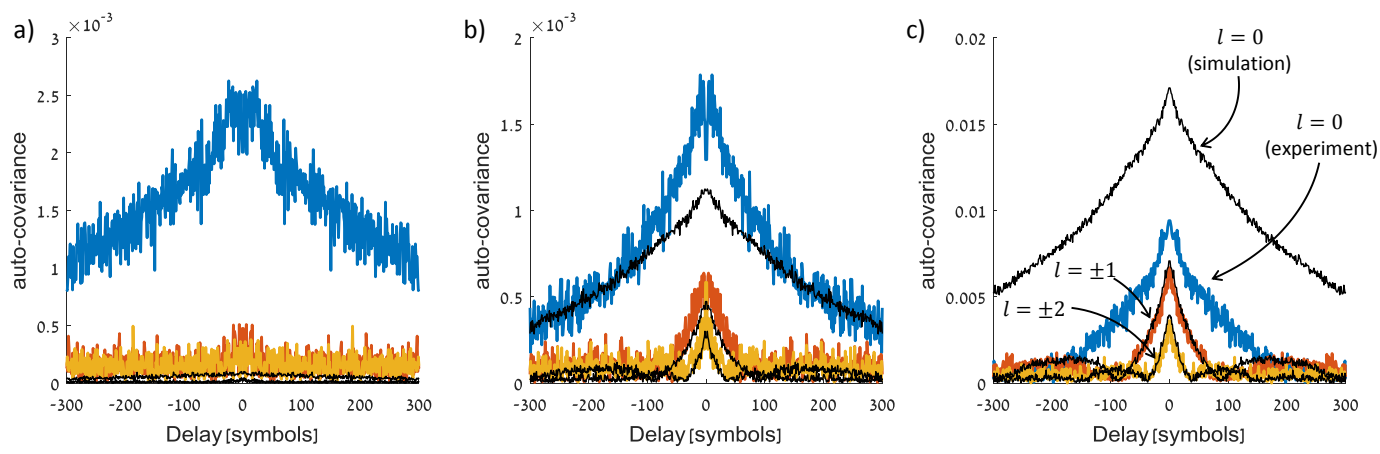

Fig. 2: Example of measured ACF of the top diagonal element of $\mathbf{R}_{l}^{(n)}$, for $l=0$ (blue), $l=1$ (red), and $l=2$ (yellow). Launch powers are $-6.5 \mathrm{dBm}(\mathrm{a}),-0.5 \mathrm{dBm}(\mathrm{b})$, and $5.5 \mathrm{dBm}(\mathrm{c})$. Black curves were obtained in split-step simulations. The large discrepancy in $l=0$ is due to laser phase-noise and phase recovery.

ery algorithm, which were not present in the simulations.

In Fig. 2a, transmission is essentially linear and the $l= \pm 1, \pm 2$ terms are very small as expected (here the measured $l=0$ contribution is totally due to laser phase-noise remaining after phase recovery). When the power increases (Figs. 2b and 2c), the effect of nonlinearity becomes evident and the contribution of the $l= \pm 1, \pm 2$ terms increases as theoretically predicted. The odd reduction in phase noise between Figs. $2 a$ and $2 b$ is probably due to different behavior of the carrier phase estimation algorithm at different OSNR levels. In Fig. 3 we show the correlation lengths of the various ISI terms (Fig. 3a) and their normalized variances (Fig. 3b). While all quantities were assessed in the high power regime where the error-bars are smallest, we note that neither the ratio between variances, nor the correlation lengths depend on the launch power. Although the correlation length reduces with ISI order, the terms $l= \pm 1, \pm 2, \pm 3$ remain correlated over notable time durations, suggesting that their equalization may be feasible. In addition, as seen in Fig. 3b, the summed variance of $l= \pm 1, \pm 2, \pm 3$ is larger than the variance of $l=0$, suggesting that the benefit of properly optimized equalization ${ }^{4}$ should be notable.
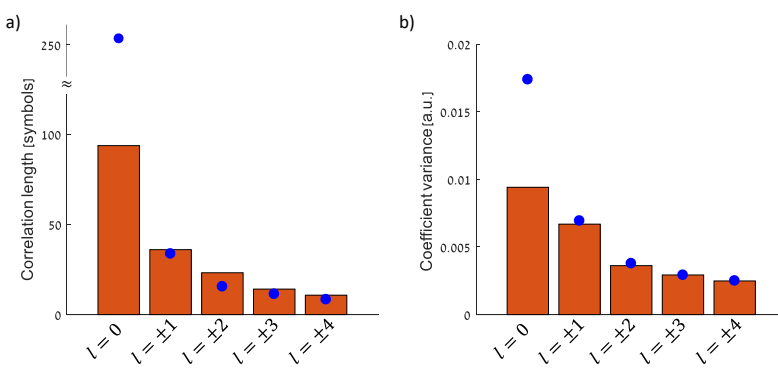

Fig. 3: Correlation lengths (a) and normalized variances (b) of top diagonal ISI elements. Circles show simulation results and bars show measurement results.

\section{Conclusions}

We proposed a method for experimentally characterizing the time varying ISI process produced by XPM-induced NLIN. We showed the existence of long temporal correlations for higher than zero order ISI terms, suggesting that their combined effect (which is comparable to that of phase-noise) is also addressable by adaptive equalization, enabling more effective nonlinearity mitigation.

\section{Acknowledgements}

TAU acknowledge financial support from ISF grant number 1401/16. UCL acknowledge financial support from the UK EPSRC under the UNLOC Programme Grant EP/J017582/1

\section{References}

[1] R. Dar et al., "Accumulation of nonlinear interference noise in fiber-optic systems," Opt. Express, 22, 12, (2014).

[2] M. Secondini et al., "Analytical fiber-optic channel model in the presence of cross-phase modulation," Photon. Technol. Lett., 24, 22, (2012).

[3] O. Golani et al., "Modeling the bit-error-rate performance of nonlinear fiber-optic systems," J. Lightwave Technol., 34, 15, (2016).

[4] O. Golani et al., "Kalman-mlse equalization of nonlinear noise," In OFC 2017, W2A-46.

[5] M. Secondini and E. Forestieri, "On XPM mitigation in WDM fiber-optic systems," Photon. Technol. Lett., 26, 22, (2014).

[6] M. Yankov et al., "Nonlinear phase noise compensation in experimental WDM systems with 256QAM," J. Lightwave Technol., 35, 8, (2017).

[7] D. Zibar et al., "Machine learning techniques in optical communication," J. Lightwave Technol., 34, 6, (2016).

[8] R. Dar et al., "Inter-channel nonlinear interference noise in WDM systems: modeling and mitigation," J. Lightwave Technol., 33, 5, (2015).

[9] T. Fehenberger et al., "Experimental analysis of correlations in the nonlinear phase noise in optical fiber systems," In ECOC 2016.

[10] P. Poggiolini and Y. Jiang, "Recent advances in the modeling of the impact of non-linear fiber propagation effects on uncompensated coherent transmission systems," J. Lightwave Technol., 35, 3, (2017).

[11] C. Schmidt-Langhorst et al., "Experimental analysis of nonlinear interference noise in heterogeneous flex-grid WDM transmission," In ECOC 2015

[12] P. W. Berenguer, et al., "Nonlinear Digital Pre-distortion of Transmitter Components," J. Lightwave Technol., 34, 8, (2016). 